Dansk Ultralyddiagnostisk Selskab

\title{
Intestinal ultrasound in ulcerative colitis - an objective biomarker for treatment response?
}

\section{Johan F.K.F. Ilvemark, MD, PhD fellow.}

Department of Gastroenterology, Herlev Hospital, University of Copenhagen, Copenhagen, Denmark.

The use of transabdominal intestinal ultrasound (IUS) as an objective biomarker of inflammation in ulcerative colitis (UC) is gaining acceptance [1]. IUS ability to correctly identify inflammation and extent of inflammation has been explained in several studies [2]. The advantages over endoscopy or other cross-sectional modalities are many; IUS is a non-invasive, preparation - and radiationfree, easily repeated and patient friendly modality [1]. When visualizing disease burden in real-time, clinicians can better explain and motivate treatment decisions, helping patients to understand their disease [3].

Different IUS parameters of inflammation (increased bowel wall thickness (BWT), in- creased color doppler signals, disrupted bowel wall stratification, loss of haustration, presence of inflammatory mesenteric fat, ascites and mesenteric lymphadenopathy) has been shown to significantly improve/restore during treatment [4]. BWT is the most common and examined parameter [2]. In the largest UC follow-up study to date $(n=224)$, the percentage of patients with an increased BWT as baseline (sigmoid colon $89 \%$ and descending colon $83 \%$ ), decreased significantly already within the first 2 weeks of treatment (sigmoid colon $38 \%$ and descending colon $43 \%$ ). BWT remained low at week 6 and 12 , with a high correlation with clinical response at week 12 [4]. It has therefore been suggested, that IUS could be an objective biomarker for treatment response in ulcerative colitis and a predicter of treatment outcome. Several studies are currently examining this hypothesis.
IUS might be proven especially useful in severe UC. Severe UC patients has a $34 \%$ risk of steroid treatment failure [6], which is associated with a higher mortality rate compared to steroid treatment responders (2.9-1.8\% vs. $0.19-0.0 \%$ [7]. Early identification of treatment failure is therefore highly warranted. Smith et al. recently published a pilot study, consisting of 10 patients hospitalized with severe UC. A high BWT (median $6.2 \mathrm{~mm}$ compared to $4.6 \mathrm{~mm}$ ) assessed within the first 24 hours of admission, was predictive of steroid treatment failure [5].

Our research group (Herlev, Hvidovre and Aarhus University Hospital, Denmark), has recently finished recruiting 60 hospitalized UC patients. Before intravenous steroid treatment, all patients underwent an IUS assessment (based on BWT, color doppler signals, disrupted bowel wall stratification, loss of haustration, and presence of inflam-

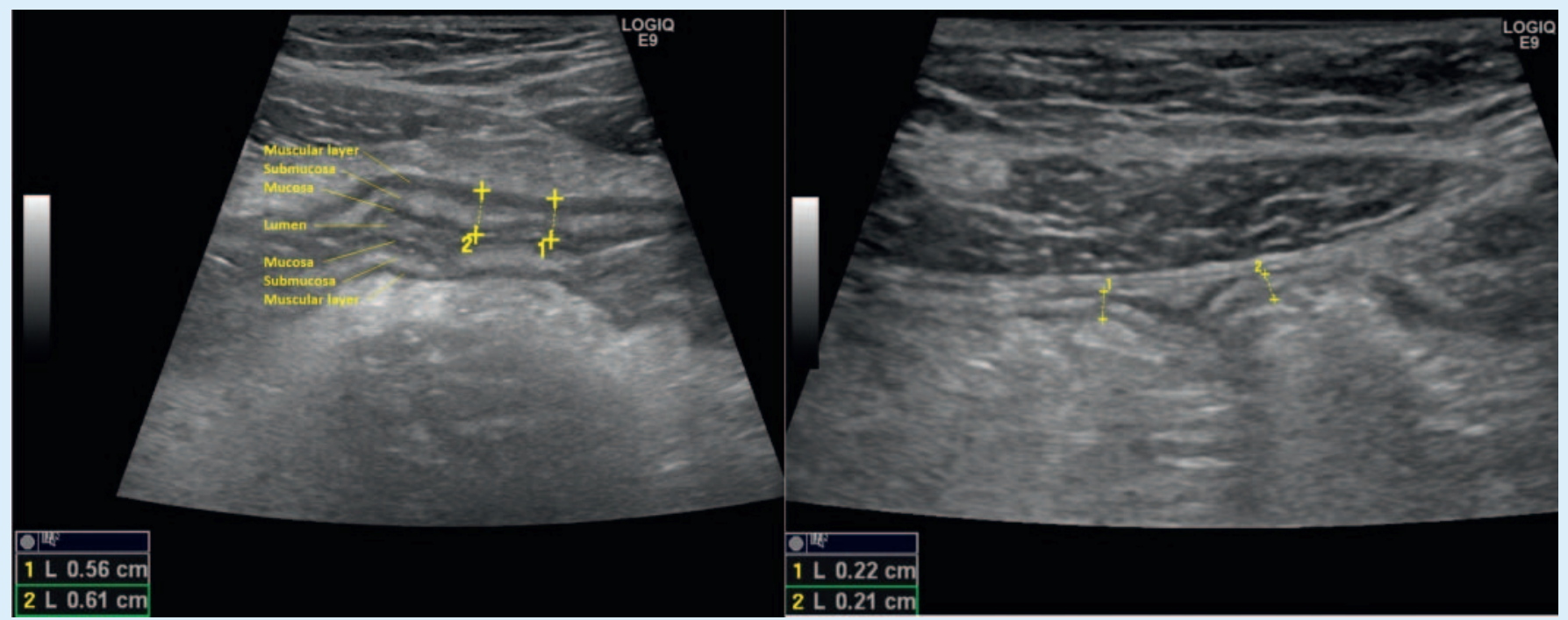

- Fig. 1 Left - Sigmoid colon before treatment (Bowel wall thickness pathological, >3 mm). Right - Sigmoid colon after treatment (Bowel wall thickness normal, $\leq 3 \mathrm{~mm}$ ). 
matory mesenteric fat), followed by a follow-up scan at $48 \pm 24$ hours after treatment initiation. We hypothesize, that IUS can already detect treatment response within the first $48 \pm 24$ hours. Data is currently being analyzed.

In conclusion, the use of transabdominal intestinal ultrasound as an objective biomarker for treatment response in UC is gaining acceptance. Initial data are promising, while more studies are highly warranted.

\section{References}

[1] Bryant RV, Friedman AB, Wright EK et al. Gastrointestinal ultrasound in inflammatory bowel disease: An underused resource with potential paradigm-changing application. Gut 2018: 973-985. doi:10.1136/gutjnl-2017-315655

[2] Smith RL, Taylor KM, Friedman AB et al. Systematic Review: Clinical Utility of Gastrointestinal Ultrasound in the Diagnosis, Assessment and Management of Patients With Ulcerative Colitis. J Crohn's Colitis 2020; 14: 465-479. doi:10.1093/ecco-jcc/jjz163

[3] Rajagopalan A, Sathananthan D, An YK et al. Gastrointestinal ultrasound in inflammatory bowel disease care: Patient perceptions and impact on disease-related knowledge. JGH Open 2020; 4: 267-272. doi:10.1002/ jgh3.12268

[4] Maaser C, Petersen F, Helwig U et al. Intestinal ultrasound for monitoring therapeutic response in patients with ulcerative colitis: Results from the TRUST\&UC study. Gut 2020; 69: 1629-1636. doi:10.1136/gutjnl-2019-319451
[5] Smith RL, Taylor KM, Friedman AB et al. Early Assessment With Gastrointestinal Ultrasound in Patients Hospitalised for a Flare of Ulcerative Colitis and Predicting the Need for Salvage Therapy: A Pilot Study. Ultrasound Med Biol 2021; 47: 1108-1114. doi:10.1016/j.ultrasmedbio.2020.12.001

[6] Grant RK, Jones GR, Plevris N et al. The ACE (Albumin, CRP and Endoscopy) Index in Acute Colitis: A Simple Clinical Index on Admission that Predicts Outcome in Patients With Acute Ulcerative Colitis. Inflamm Bowel Dis 2021; 27 : 451-457. doi:10.1093/ibd/izaa088

[7] Lynch RW, Lowe D, Protheroe A et al. Outcomes of rescue therapy in acute severe ulcerative colitis: data from the United Kingdom inflammatory bowel disease audit. Aliment Pharmacol Ther 2013; 38: 935-945. doi:10.1111/apt.12473 